\title{
When is a quantity additive, and when is it extensive?*
}

\author{
Hugo Touchette ${ }^{\dagger}$ \\ Department of Physics and School of Computer Science, \\ McGill University, Montréal, Québec, Canada H3A 2A7
}

October 23, 2018

\begin{abstract}
The difference between the terms additivity and extensivity, as well as their respective negations, is critically analyzed and illustrated with a few examples. The concepts of subadditivity, pseudo-additivity, and pseudo-extensivity are also defined.
\end{abstract}

To say that a given quantity (physical or mathematical) is additive and to say that a quantity is extensive are two different affirmations which, unfortunately, appear too often as undifferentiated in the physics literature. The assimilation of the two terms is especially present in studies related to the entropy measure of Tsallis [1], and to the so-called field of non-extensive thermostatistics or Tsallis' statistics which is based on this measure of entropy [2]. In these studies, it is not uncommon to see the words additivity and extensivity being used as synonymous, and to read sentences such as "...the appropriate framework to describe non-extensive behavior is Tsallis' statistics, because of the non-additivity property of Tsallis' entropy.” But, how exactly is the non-additivity property of Tsallis' entropy related to non-extensivity? Are these concepts linked together simply because they are thought to mean the same thing?

These questions are raised not with the intention of criticizing the results related to nonextensive statistics; what is more important is the fact that they point to a somewhat misleading and careless usage of scientific jargon. That this carelessness persists would not by itself be so problematic, were it not for the fact that the difference between additivity and extensivity is at the very root of the issues raised by non-extensive statistics. For this reason, it seems more than advisable to rehabilitate the proper meaning of these two terms by reviewing their

\footnotetext{
${ }^{*}$ Contribution to the Proceedings of the International School and Conference on Non-Extensive Thermodynamics and Physical Applications, Villasimius, Sardegna, Italy, May 2001.

†E-mail addresses: htouc@cs.mcgill.ca, htouchet@alum.mit.edu (H. Touchette).
} 
standard 'textbook' definitions. This is the purpose of the present contribution. Of course, in doing so, no new results are likely to be conveyed; however, it can only be at our advantage to repeat a number of concepts and definitions which are fundamental to statistical physics. As Wittgenstein once wrote: "what can be said at all, can be said clearly." After reading this short review, it is hoped that researchers will follow this advice, and will indeed be more perspicuous in discussing any subject which connects itself to additivity and extensivity.

To begin this review, let us fix the context of the discussion. The qualifiers 'additive' and 'extensive' apply to systems which are composite in the sense that they are composed of many components or subsystems. A typical example of such composite systems is a gas composed of many identical particles which may or may not interact with each others. If we label these particles by the index $i=1,2, \ldots, n$, and represent the state of each of these particles by using the variable $x_{i}$, then the possible interactions which may exist between the $n$ particles can be modeled either in the form of a total energy or Hamiltonian function $U\left(x^{n}\right)$ associated with the joint state $x^{n}=x_{1} x_{2} \ldots x_{n}$ of the complete system, or, more generally, by a joint probability distribution $p\left(x^{n}\right)$ which gives the probability that the particle 1 of the gas is in the state $x_{1}$, particle 2 in $x_{2}$, and so on.

Now, suppose that we are interested in calculating or in measuring physically a quantity (an observable) which is a function, say $Q\left(x^{n}\right)$, of the joint state of a composite system. For this quantity, we may ask: can $Q\left(x^{n}\right)$ be evaluated with the recourse of terms of order lower than $n$, i.e., with terms like $Q\left(x^{m}\right)$, where $x^{m}$ is any $m$-particles $s u b$-state of $x^{n}$ with $m<n$ ? In particular, can it be decomposed as the sum of the $Q\left(x_{i}\right)$ 's? The concept of additivity partly classifies, as follows, the answers that these two questions can receive.

Additivity. A many-body (or joint) physical observable $Q\left(x^{n}\right)$ is said to be additive with respect to two subsystems with states $x^{m}=x_{1} x_{2} \ldots x_{m}$ and $x^{n-m}=$ $x_{m+1} x_{m+2} \ldots x_{n}$ if $Q\left(x^{n}\right)=Q\left(x^{m}\right)+Q\left(x^{n-m}\right)$.

Obviously, this definition can be generalized to any partitions of the joint state $x^{n}$ in two or more subsystems. As a result, we have the following stronger version of additivity.

Complete additivity. $Q\left(x^{n}\right)$ is said to be completely additive if it is the sum of the one-particle (or marginal) contributions to $Q$, i.e., if $Q\left(x^{n}\right)=\sum_{i=1}^{n} Q\left(x_{i}\right)$.

A first example of a completely additive quantity which immediately comes to mind is the total energy of a set of non-interacting bodies (e.g., the particles of an ideal gas). 'Noninteracting', in this case, can in fact be taken to be equivalent to 'additive energy', because, as we all know, interactions appear in the expression of the total energy as extra irreducible energy or potential terms which, in effect, allow a finite number of bodies to exchange energy. These terms are irreducible in the sense that they are themselves non-additive.

In the case where $p\left(x^{n}\right)$ is given instead of $U\left(x^{n}\right)$, a similar characterization of the properties of $p\left(x^{n}\right)$ is possible if one interprets the logarithm of the inverse of this quantity as 
some kind of energy $\mathcal{U}\left(x^{n}\right)$, or pseudo-energy in order to distinguish it from the 'real' energy $U\left(x^{n}\right)$. This is the basis of the so-called thermodynamic formalism of stochastic systems [3, 4, 5]. In short, if $p\left(x^{n}\right)$ is factorizable or separable, i.e., if it can be expressed as the product $p\left(x^{n}\right)=p\left(x_{1}\right) p\left(x_{2}\right) \cdots p\left(x_{n}\right)$ of marginal probability distributions, then the corresponding pseudo-energy is additive:

$$
\mathcal{U}\left(x^{n}\right)=-\ln p\left(x^{n}\right)=-\sum_{i=1}^{n} \ln p\left(x_{i}\right)=\sum_{i=1}^{n} \mathcal{U}\left(x_{i}\right) .
$$

On the other hand, when $p\left(x^{n}\right)$ is non-factorizable, $\mathcal{U}\left(x^{n}\right)$ becomes non-additive. Indeed, in this latter case, $p\left(x^{n}\right)$ can only be written generically as a product of conditional probabilities of the form

$$
p\left(x^{n}\right)=p\left(x_{1}\right) p\left(x_{2} \mid x_{1}\right) p\left(x_{3} \mid x_{1} x_{2}\right) \cdots p\left(x_{n} \mid x^{n-1}\right),
$$

which makes obvious that Eq.(1) is no more satisfied. In fact, each conditional probability term $p\left(x_{m} \mid x^{m-1}\right)$ above can be assimilated, again by taking the logarithm of its inverse, to an interaction pseudo-energy $\mathcal{U}\left(x^{m}\right)$ which enters in a non-additive fashion in the expression of $\mathcal{U}\left(x^{n}\right)$. This makes the connection with the properties of $U\left(x^{n}\right)$ more evident. (The connection is even more effective when both $U\left(x^{n}\right)$ and $\mathcal{U}\left(x^{n}\right)$ can be defined and related to each other, as in the case of thermodynamic systems. See Refs. [4, 5] for more details.)

Another quantity which is also completely additive for independent systems, and which is worth mentioning because of its great importance in statistical mechanics, is the joint BoltzmannGibbs-Shannon (BGS) entropy of a sequence $X^{n}=X_{1} X_{2} \ldots X_{n}$ of random variables:

$$
H\left(X^{n}\right)=-\sum_{x^{n}} p\left(x^{n}\right) \ln p\left(x^{n}\right) .
$$

By inspecting the above expression, one can see that the joint entropy $H\left(X^{n}\right)$ is nothing more than the expected total pseudo-energy (also called bit-number [4]) over all joint states $x^{n}$. As a consequence, one readily concludes that $H\left(X^{n}\right)$ is additive if and only if $p\left(x^{n}\right)$ is factorizable. As an added property, it can also be shown that $H\left(X^{n}\right)$ is strictly subadditive otherwise, which means that $H\left(X^{n}\right)<\sum_{i=1}^{n} H\left(X_{i}\right)$ when the random variables forming the state $X^{n}$ are correlated in one way or another [6]. At glance with the BGS entropy, the joint version of the entropy measure of Tsallis, given by

$$
H_{q}\left(X^{n}\right)=\frac{1}{q-1}\left(1-\sum_{x^{n}} p\left(x^{n}\right)^{q}\right)
$$

is non-additive for independent random variables. For instance, it can be verified that

$$
H_{q}\left(X_{1}, X_{2}\right)=H_{q}\left(X_{1}\right)+H_{q}\left(X_{2}\right)+(1-q) H_{q}\left(X_{1}\right) H_{q}\left(X_{2}\right)
$$


for $X_{1}$ and $X_{2}$ such that $p\left(x_{1}, x_{2}\right)=p\left(x_{1}\right) p\left(x_{2}\right)$ for all $x_{1}$ and $x_{2}$ [1]. It is precisely because of this property that Tsallis' entropy is referred to generically (but perhaps too generically) as being non-additive.

Let us now turn our attention to another aspect of composite observables which concerns their scaling properties with the number of subsystems. Given again a function $Q\left(x^{n}\right)$ of the joint state $x^{n}$, we are interested in determining if $Q\left(x^{n}\right)$ scales proportionally with $n$ or, at least, asymptotically proportionally with $n$ when this number becomes large. If it does, then $Q$ is termed extensive. More precisely:

Extensivity. A joint observable $Q\left(x^{n}\right)$ is extensive if the $Q$-density, defined by the ratio $Q\left(x^{n}\right) / n$, reaches a constant in the limit $n \rightarrow \infty$. If random variables are used, a criterion of convergence (e.g., convergence in probability, almost surely, etc.) should also be provided in order to give a meaning to the limit.

In relation to this definition, physicists commonly say that a system possesses a thermodynamic limit if its total energy and total entropy are both extensive quantities [4, 5].

At this point, it is important that the reader clearly distinguishes the concept of extensivity from that of additivity. In general, extensivity does not imply additivity, nor does additivity imply extensivity. (The latter assertion is a common pitfall.) On the one hand, there exist joint observables which are non-additive, but which are nonetheless extensive. The joint BGS entropy of ergodic sequences of correlated random variables is often cited as an example of a quantity which is non-additive, but which converges in density to a constant known as the entropy rate. (See [5, 6] for a definition of ergodic stochastic processes, and [6] for a description of entropy rates.) On the other hand, a quantity may well be additive without being extensive. For instance, it is not so much difficult to construct a system composed of independent particles characterized by a total additive energy $U\left(x^{n}\right)$ which has no limits in $n$. But, admittedly, this would be a very peculiar and exceptional example of an additive yet non-extensive quantity, since, in most cases encountered in physics, additivity does imply extensivity. Furthermore, in many models of physical systems, the fact that a quantity $Q$ is non-extensive is intimately related to the fact that it is non-additive, for it is usually the non-additive part of this quantity which divergences as $n \rightarrow \infty$, and which thus prevents the $Q$-density to converge. Such a singularity occurs, for instance, when the interaction energy or pseudo-energy between subsystems is longrange in the sense that it decays very slowly with the distance (if we are concerned with particles in space) or with the neighborhood separation (as for spin-lattice systems). The dimensionality of the system considered plays also an important role in defining what 'long-range' means (see, e.g., [级, 5]).

By way of conclusion, note that it is sometimes possible to transform a non-extensive quantity into an extensive one by rescaling it using a volume $(n)$ dependent factor or, simply, by dividing the quantity in question by its nonlinear scaling law if it is characterized by such a law (Kac's prescription [7]). The 'renormalized' quantity which results from this procedure 
is called pseudo-extensive (see [8] for a specific example). On a related note, a non-additive quantity $Q\left(x^{n}\right)$ may be written in a pseudo-additive way if one can find another quantity $\tilde{Q}\left(x_{i}\right)$ such that

$$
Q\left(x^{n}\right)=\sum_{i=1}^{n} \tilde{Q}\left(x_{i}\right),
$$

with the additional requirement that $\tilde{Q}\left(x_{i}\right)=Q\left(x_{i}\right)$ for all $i$ whenever $Q\left(x^{n}\right)$ is additive. This last concept of pseudo-additivity is probably not well-known to many; yet, it is effectively in use when one expresses the total energy of a gas of electrons in a semiconductor as a sum of free electron energies with a different renormalized or effective mass factor. At the level of the entropy, a related procedure has also been described recently by which the joint BGS entropy $H\left(X^{n}\right)$ of an ergodic source of correlated random variables can be transformed in an pseudo-additive fashion as the sum of the marginal Tsallis' entropies $H_{q}\left(X_{i}\right), i=1,2, \ldots, n$, all assuming the same fixed value of $q \geq 1$ [ [9]. This opens up, perhaps surprisingly, the possibility of applying Tsallis' entropy to a wider class of systems than previously thought, and, particularly, to the class of fully extensive systems which have been hitherto neglected by the community of researchers working on Tsallis' statistics.

\section{Acknowledgments}

I wish to thank the organizers of the NEXT2001 Conference for their application in making the conference a pleasant and fruitful meeting, and for having provided the financial support which enabled me to cross the Atlantic Ocean. This work was sponsored in part by NSERC (Canada), and FCAR (Québec).

\section{References}

[1] C. Tsallis, J. Stat. Phys. 52 (1988) 479.

[2] S. Abe, Y. Okamoto, Nonextensive Statistical Mechanics and Its Applications, Springer Verlag, New York, 2001.

[3] D. Ruelle, Thermodynamic Formalism: The Mathematical Structures of Classical Equilibrium Statistical Mechanics, Addison-Wesley, Reading, Mass., 1978.

[4] C. Beck, F. Schlögl, Thermodynamics of Chaotic Systems: An Introduction, Cambridge University Press, Cambridge, 1993.

[5] R. Badii, A. Politi, Complexity: Hierarchical Structures and Scaling in Physics, Cambridge University Press, Cambridge, 1997.

[6] T.M. Cover, J.A. Thomas, Elements of Information Theory, John Wiley, New York, 1991. 
[7] J. Barr, D. Mukamel, S. Ruffo, Phys. Rev. Lett. 87 (2001) 030601, and references therein.

[8] C. Anteneodo, C. Tsallis, Phys. Rev. Lett. 80 (1998) 5313.

[9] H. Touchette, Talk at the NEXT2001 Conference. Full paper in preparation. 